\title{
Design and Development of Polyaniline-coated Fabric Strain Sensor for Goniometry Applications
}

\author{
N. Muthukumar \\ Department of Textile \\ Technology, \\ PSG College of Technology, \\ Coimbatore-641004, India
}

\author{
G. Thilagavathi \\ Department of Textile \\ Technology, \\ PSG College of Technology, \\ Coimbatore-641004, India
}

\author{
T.Kannaian \\ Department of Electronics, \\ PSG College of Arts \&Science, \\ Coimbatore-641014, India
}

\begin{abstract}
In the last few years, the smart textile area has become increasingly widespread, leading to developments in new wearable sensing systems. As conventional sensor techniques often cause problems for long term patient monitoring (e.g. skin irritation, hampering wires), elegant solutions are explored to integrate sensors in clothing. By using the textile material itself as a sensor, the integration is increased resulting in even more patient friendliness.

In this paper, a flexible fabric strain sensor with high sensitivity, good stability and large deformation is reported. It is fabricated by in-situ polymerization of polyaniline on the fabric substrate at low temperature. Thickness and morphology of the conducting thin film on the surface of the fibers were examined by scanning electron microscopy (SEM). The resistivity of the PANi coated fabric was measured using standard two probe apparatus.

The measurement of the conductivity change with strain shows that the fabrics so prepared exhibits a high strain sensitivity while its good stability is indicated by a small loss of conductivity after the thermal and humidity aging tests, and supported by the slight change in conductivity over storage of 90 days. The developed flexible strain sensor can be used in the preparation of smart garment for goniometry applications.

Keywords: conductivity, goniometry, fabric strain sensor, polyaniline, sensitivity
\end{abstract}

\section{INTRODUCTION}

Inherently conducting polymers such as polypyrrole and polyaniline are often referred to as a "synthetic metals", which possesses the electrical and magnetic properties of a metal, while retaining the mechanical properties of a polymer. Active research has been carried out to investigate the application of these materials in corrosion protection, rechargeable batteries, electrochromic displays, conducting composite materials, biosensors, chemical gas sensors, actuators, microextraction platforms, electronics, electrochemical energy sources, optical devices and smart fabrics.

Electro-textiles can be defined as textiles with unobtrusively built-in electronic and photonic functions. They are mostly used for electromagnetic shielding, anti-static and heating purposes, and also for soft circuits: electric circuits or sensors made out of a combination of special fabrics, threads, yarns and electronic components. Electrical functions can be embedded in textiles by using weaving, knitting and embroidery or nonwoven production techniques. The integration of electronic properties directly into the clothing environment carries some advantages such as increased comfort, mobility, usability and aesthetic properties. However, there are some challenges to be addressed. Yarns that are used for making cloth should be fine and elastic in order to ensure the wearer's comfort. The fibres have to be able to withstand handling and fabrics should have low mechanical resistance to bending and shearing which means they can be easily deformed and draped.

The creation of textile-based strain sensors has attracted researchers' attention so many investigators have studied this area and numerous different kinds of technique have been used in order to create strain sensing structures. These sensors have been used to measure human body movements or respiratory activity [1\&2]. De Rossi et al. [3] created strain sensing fabrics by coating Lycra/cotton fabrics with polypyrrole and carbon loaded rubbers. Polypyrrole-coated fabrics showed an average gauge factor of about -13 . These strain sensing fabrics exhibited a strong variation of strain-resistance with time and they showed a high response time to applied mechanical stimulus. Fabrics coated with carbon loaded rubber had a gauge factor of approximately 2.5 and fabric sensors made with this type of material showed good strain sensing properties between $1 \%$ and $13 \%$ strain. Xue et al. [4] also created strain sensing structures by coating nylon 6 and polyurethane fibres with polypyrrole. According to this research, polypyrrole-coated nylon 6 fibres showed good sensing performance, whereas polypyrrole-coated polyurethane fibres did not produce promising results as a strain sensing structure. Also, Mattmann et al. [5] created a strain sensor by using a thermoplastic elastomer and carbon particles and they were able to recognize upper body postures with an accuracy of $97 \%$.

We report a PANi coated flexible strain sensor which was prepared by in-situ polymerization of polyaniline on the fabric substrate at low temperature. The sensor so prepared fabricated shows both high strain resistivity for a large deformation and a good environmental stability.

\section{EXPERIMENTAL PROCEDURE}

\subsection{Materials}


Aniline,

concentrated

$\mathrm{HCl}$,

and Ammoniumpersulfate (APS), all of AR grade and obtained from S.D. Fine Chemicals Ltd., India, were used. Aniline was distilled twice before use. 92\% nylon and $8 \%$ lycra single jersey fabric was used. The fabric specifications are, Thickness: $0.573 \mathrm{~mm}$, GSM: 200grams, wales per inch (WPI): 64, course per inch(CPI): 88 .

\subsection{Synthesis of conductive fabrics}

Conductive fabrics were developed by in situ chemical polymerization of aniline on the fabrics. In this process, freshly distilled $0.5 \mathrm{M}$ aniline was dissolved in the bath containing $0.35 \mathrm{~N}$ HCL solution for diffusion. A vigorous stirring was given to the bath containing mixtures of aniline and aqueous acid to attain the homogeneous mixing. Dry preweighed fabric sample was placed in the above solution at $40^{\circ} \mathrm{C}$ and allowed for $2 \mathrm{~h}$ to soak well with the monomer and dopant solution. $0.25 \mathrm{M}$ ammonium per sulfate was separately dissolved in $0.35 \mathrm{~N}$ HCL solution for polymerization. The aqueous oxidizing agent in the separate bath was then slowly added in to the diffusion bath to initiate the polymerization reaction. The oxidant to aniline ratio was kept at around 1.25. The whole polymerization reaction was carried out at $5^{\circ} \mathrm{C}$ for 1hour. After completing the polymerization process, the coated fabric was taken out and washed in distilled water containing $0.35 \mathrm{~N}$ HCL and dried at $60{ }^{\circ} \mathrm{C}$ [6]. The SEM images were recorded by JEOL SEM (model JSM- 6360) to study the surface morphology of the control and polyaniline treated samples in the longitudinal view.

\subsection{Electrical resistance measurements}

Electrical resistance measurements were performed on all samples after conditioning the samples in a standard atmosphere. The resistance was measured ten times on each side of the sample and the average values were taken. The American Association of Textile Chemists and Colourists (AATCC) test method 76-1995 was used to measure the resistance of the samples and the surface resistivity of the fabric was calculated as follows

$$
R=R_{s}(l / w)
$$

where $R$ is the resistance in ohms; $R_{s}$, the sheet resistance or surface resistivity in ohms/square; $l$, the distance between the electrodes; and w, the width of each electrode

\subsection{Test Procedure for Knitted Strain Sensors}

The PANi coated conductive nylon lycra fabric having size of $150 \mathrm{~mm} \times 30 \mathrm{~mm}$ was extended with help of fabric extension meter from an initial length of $15 \mathrm{~cm}$ and then they were extended for $50 \%$ i.e. $8 \mathrm{~cm}$ at intervals of $1 \mathrm{~cm}$. The resistance change thus caused due to the extension was measured using Waynekerr 4321 LCR Meter. The extension level of $50 \%$ was chosen to mirror typical human body extensions, as the proposed sensor can be used for monitoring human body movements. The sample was measured five times and the relaxation between measurements was $10 \mathrm{sec}$.

In strain sensors, the gauge factor (GF) is an important parameter and it gives information about the sensitivity of the sensor. The GF is calculated as follows:
$\mathrm{GF}=\Delta \mathbf{R}$

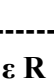
Where:
$\Delta \mathrm{R}=$ the change in the resistance;
$\mathrm{R}=$ the initial resistance (the resistance before extension);
$\varepsilon=$ the strain value.

\subsection{Experimental set up for Joint Angle Measurement}

The PANi coated conductive nylon lycra fabric having size of $150 \mathrm{~mm} \times 30 \mathrm{~mm}$ was attached to an elbow sleeve using a Velcro as shown in Figure 1a. The sleeve which is integrated with sensors was fixed in a subject arm as shown in Figure $1 \mathrm{~b}$ for the measurement of resistance change for angular displacement. The arm was moved for different angle positions with use of a mechanical goniometer and corresponding resistance change was noted using Waynekerr 4321 LCR Meter. Three trials were taken and corresponding readings has been recorded. Then, the angles were converted into equivalent resistance and calibration done. Hence, the resistance change can be measured for further use and can be converted into angles based on the calibration [7].

\subsection{Aging behavior Measurement}

Both thermal and humidity aging tests were carried out by recording the conductivity change of the PANi-coated fabric put in a Programmable Environmental Test Chamber (Remi Instrument Ltd, Mumbai), where both the temperature and humidity can be controlled. For the thermal aging, the humidity is always kept at $65 \% \mathrm{RH}$. The temperatures investigated include $20,35,50$ and $60^{\circ} \mathrm{C}$ and the temperature changes every two hours. The humidity aging was carried out at $30^{\circ} \mathrm{C}$. The humidities investigated include 40, 55, 70 and $90 \%$ RH and the humidity changes every two hours [8].

\section{RESULTS AND DISCUSSION \\ 3.1 SEM Studies}

The surface views of SEM micrographs of the control nylon lycra fabric and nylon lycra + PANI are shown in Figure 2. Just a glance at the fabric with the naked eye shows a uniform color (in this case, green), indicating that PANi has penetrated into the fabric. However, the SEM studies reveal how evenly the surface has been coated as well as the depth of penetration.

From the SEM studies, it is clear that the PAN particles are very evenly deposited on the fabric, and are seen as small globules. The surface studies clearly reveal uniform distribution even at the microscopic level, which is necessary for the reproducibility and reliability of applications. The 


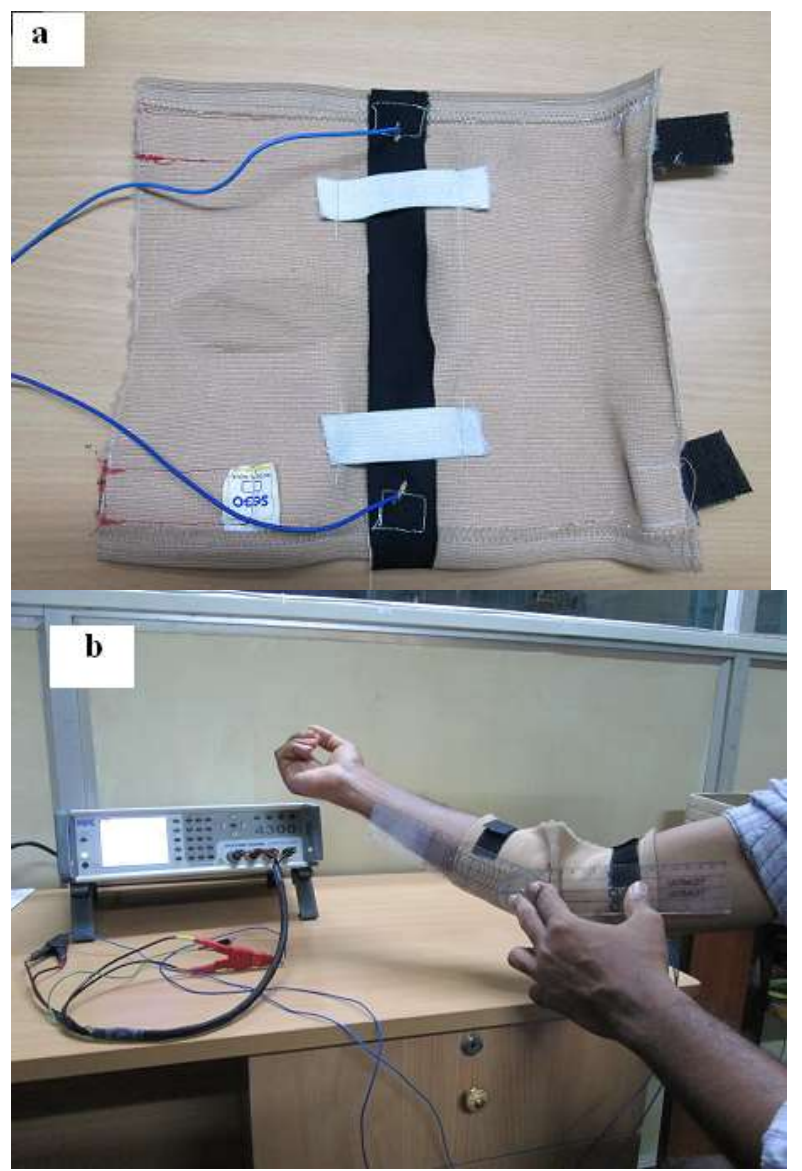

Figure 1 a) Sensor Integrated Elbow Sleeve b) Elbow angle measurement using developed sensor

diffusion and polymerization of aniline in the fabric is evident at the macroscopic level in terms of the increased thickness of the fabric, from $0.573 \mathrm{~mm}$ to $0.575 \mathrm{~mm}$. The fibres are swelled due to polyaniline impregnation. Because of this swelling of fibres, the thickness of PANI coated fabrics increases.

\subsection{Electrical properties}

Surface resistivity is a material property that is normally considered constant and ideally independent of measurement technique. Surface resistivity measurement is often used to characterize fabric resistivity and is typically reported as ohm/square. We studied the electrical resistivity of the polyaniline coated fabric by two probe resistivity measurement in a normal environment at $65 \% \mathrm{RH}$. The surface resistivity value of the fabrics coated with polyaniline is $3.5 \mathrm{~K} \mathrm{ohm} /$ square.

\subsection{Electrical resistivity for linear extension}

The PANi coated nylon lycra fabric sample was extended up to $50 \%$ extension and the changes in resistance with extension were noted and are as shown in the Table 1 and Figure 3. The fabric sensor has negative resistance versus elongation change effect, which means that the resistance decreases with extension. The reason for that is the
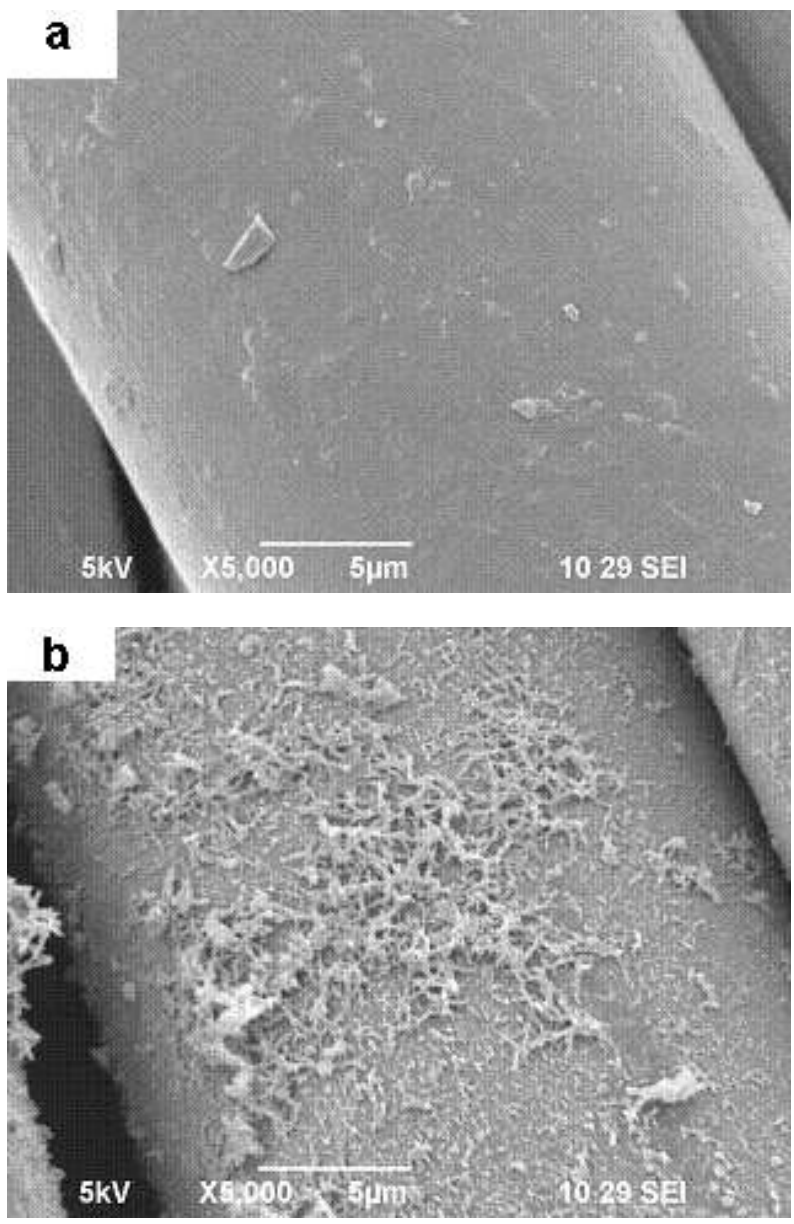

Figure 2 a) control nylon lycra fabric b) PANi treated nylon lycra fabric

construction of the sensor. When the sample is stretched, the course and wale spacing decreases, higher contact pressure occurs between adjacent course and wales. The conductive connections increase and the resistance decreases. The developed fabric strain sensor has the average gauge factor of 0.92 .

According to Holm's contact theory:

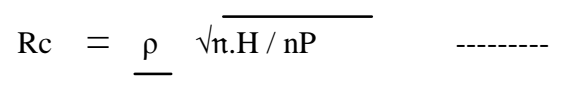

2

Where:

$$
\begin{aligned}
& \mathrm{Rc}=\text { contact resistance; } \\
& \rho=\text { electrical resistivity; } \\
& \mathrm{H}=\text { material hardness; } \\
& \mathrm{n}=\text { number of contact points; } \\
& \mathrm{P}=\text { contact pressure }
\end{aligned}
$$


International Journal of Science and Engineering Applications

Special Issue NCRTAM ISSN-2319-7560 (Online)

Table 1: Electrical resistance changes in ohm with the elongation of PANi coated fabrics

\begin{tabular}{|c|c|c|c|c|c|c|c|c|c|c|}
\hline \multirow{2}{*}{$\begin{array}{l}\text { Length } \\
\text { in } \mathrm{cm}\end{array}$} & \multicolumn{2}{|c|}{ Trial 1} & \multicolumn{2}{|c|}{ Trial 2} & \multicolumn{2}{|c|}{ Trial 3} & \multicolumn{2}{|c|}{ Trial 4} & \multicolumn{2}{|c|}{ Trial 5} \\
\hline & Extension & Recovery & Extension & Recovery & Extension & Recovery & Extension & Recovery & Extension & Recovery \\
\hline 15 & 22.31 & 24.124 & 24.088 & 24.836 & 24.038 & 24.87 & 22.97 & 25.161 & 23.455 & 25.068 \\
\hline 16 & 21.146 & 22.044 & 21.302 & 23.557 & 21.519 & 22.67 & 21.239 & 22.758 & 21.575 & 23.023 \\
\hline 17 & 19.457 & 20.844 & 20.352 & 22.438 & 19.801 & 20.827 & 19.702 & 21.234 & 20.017 & 21.822 \\
\hline 18 & 18.434 & 19.285 & 19.055 & 20.045 & 18.759 & 19.639 & 18.53 & 19.937 & 18.902 & 20.367 \\
\hline 19 & 17.585 & 18.67 & 17.953 & 19.732 & 17.633 & 18.92 & 17.642 & 19.084 & 18299 & 19.693 \\
\hline 20 & 16.74 & 17.767 & 17.018 & 18.497 & 17.082 & 17.95 & 17.175 & 18.258 & 17.584 & 18.748 \\
\hline 21 & 16.215 & 17.022 & 16.612 & 17.649 & 16.576 & 17.333 & 16.787 & 17.475 & 16.912 & 17.879 \\
\hline 22 & 15.71 & 16.156 & 16.12 & 16.628 & 16.157 & 16.469 & 16.194 & 16.717 & 16.459 & 16.913 \\
\hline 23 & 15.34 & 15.34 & 15.705 & 15.705 & 15.517 & 15.517 & 15.57 & 15.57 & 15.925 & 15.925 \\
\hline GF & \multicolumn{2}{|c|}{0.871} & \multicolumn{2}{|c|}{0.798} & \multicolumn{2}{|c|}{1.065} & \multicolumn{2}{|c|}{0.925} & \multicolumn{2}{|c|}{0.941} \\
\hline
\end{tabular}

From equation (2), it can be see that the electrical resistivity and material hardness are constant for a given material, but the number of contact points and the contact pressure are variable depending on the sensor design. Thus, higher contact pressure and increased number of contact points between conductive parts lower the contact resistance [9\&10].

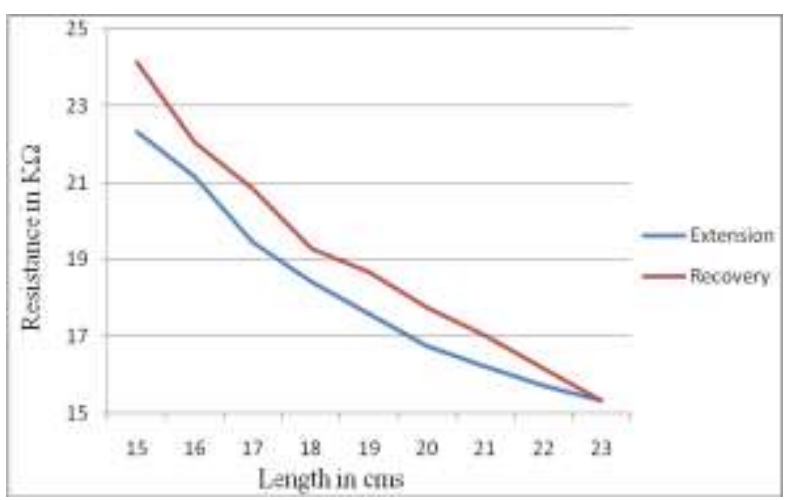

Figure 3 : Electrical resistance changes in $\mathrm{K}$ ohms with the elongation of PANi coated fabrics

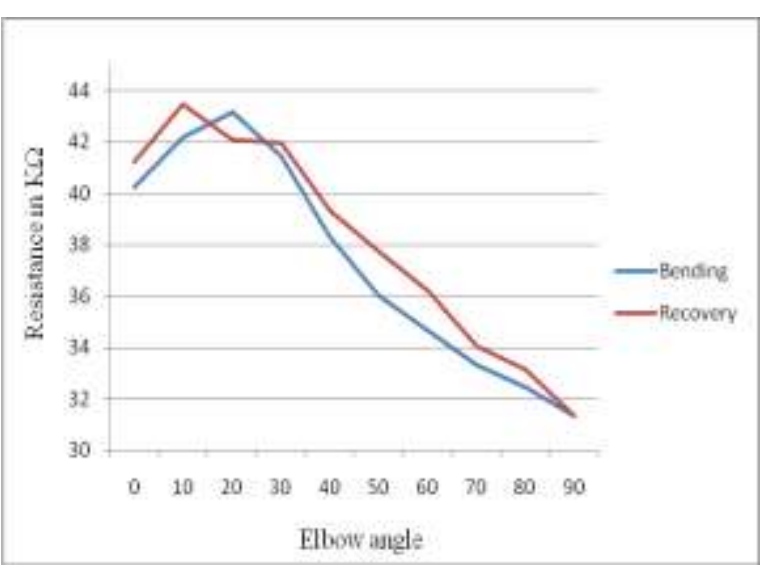

Figure 4 Change in resistance in Kohms with angular displacement

\subsection{Measurement of resistance with varying angles}

The change in resistance for various angles of movement of elbow is shown in Table 2 and Figure 4. The samples tend to have a good repeatability property for angle resistance change. There was a steady decrease in resistance with up to $90^{\circ}$ flexion of the elbow after that the change in resistance is not significant.

Table 2 Electrical resistance changes in $K$ ohms with angular displacement of PANi coated fabrics

\begin{tabular}{|l|l|l|l|l|l|l|}
\hline \multirow{2}{*}{$\begin{array}{l}\text { Angl } \\
\mathrm{e}\end{array}$} & \multicolumn{2}{|c|}{ Trial 1 } & \multicolumn{2}{c|}{ Trial 2 } & \multicolumn{2}{c|}{ Trial 3 } \\
\cline { 2 - 7 } & $\begin{array}{l}\text { Bendin } \\
\mathrm{g}\end{array}$ & $\begin{array}{l}\text { Recover } \\
\mathrm{y}\end{array}$ & $\begin{array}{l}\text { Bendin } \\
\mathrm{g}\end{array}$ & $\begin{array}{l}\text { Recover } \\
\mathrm{y}\end{array}$ & $\begin{array}{l}\text { Bendin } \\
\mathrm{g}\end{array}$ & $\begin{array}{l}\text { Recover } \\
\mathrm{y}\end{array}$ \\
\hline 10 & 41.11 & 39.658 & 43.167 & 40.65 & 42.195 & 43.466 \\
\hline 20 & 42.412 & 38.487 & 39.43 & 36.571 & 43.144 & 42.074 \\
\hline 30 & 39.347 & 36.894 & 36.337 & 35.548 & 41.449 & 41.94 \\
\hline 40 & 36.494 & 35.288 & 34.765 & 34.477 & 38.318 & 39.319 \\
\hline 50 & 34.781 & 34.485 & 33.781 & 33.67 & 36.039 & 37.749 \\
\hline 60 & 32.968 & 33.534 & 32.394 & 32.21 & 34.66 & 36.199 \\
\hline 70 & 32.345 & 32.579 & 31.213 & 31.651 & 33.331 & 34.055 \\
\hline 80 & 31.832 & 32.052 & 30.607 & 31.106 & 32.463 & 33.164 \\
\hline 90 & 31.073 & 31.073 & 30.288 & 30.288 & 31.345 & 31.345 \\
\hline
\end{tabular}

\subsection{Aging behavior}

In addition to high sensitivity, good environmental stability is especially important for the practical application. The conductivity degradation of PANi is closely related to its reaction with oxygen, especially under elevated temperatures, and moisture. Therefore, the stability of the PANi coated nylon lycra fabric was first investigated by the thermal aging and humidity aging tests. 
The thermal aging test is conducted by changing temperature from 20 to $60^{\circ} \mathrm{C}$, and the conductivity change with temperature of the PANi coated nylon lycra fabric during the thermal aging process is presented in Table. 3. It can be seen that the difference in resistance between the temperatures is quite small. In addition, at low temperature only a slight increase in resistance is observed which may be related to the stability of the dopant ion and the re arrangement of the PANi chain. Humidity can also exert an influence on the conductivity of PANi. The conductivity change with humidity of the sensor is presented in Table. 4. It was found that the humidity increase can only slightly decrease the resistance of the sensor.

Table 3 Resistance change with Temperature

\begin{tabular}{|c|c|}
\hline Temperature in ${ }^{\circ} \mathrm{C}$ & $\begin{array}{c}\text { Surface Resistance in } \\
\text { Kohm/square }\end{array}$ \\
\hline 20 & 3.8 \\
\hline 35 & 3.5 \\
\hline 50 & 3.2 \\
\hline 60 & 3.0 \\
\hline
\end{tabular}

Table 4 Resistance change with RH\%

\begin{tabular}{|c|c|}
\hline Relative Humidity in \% & $\begin{array}{c}\text { Surface Resistance in } \\
\text { Kohm/square }\end{array}$ \\
\hline 40 & 3.7 \\
\hline 55 & 3.5 \\
\hline 70 & 3.3 \\
\hline 90 & 3.2 \\
\hline
\end{tabular}

The thermal and humidity aging tests show that the conductivity of the strain sensor from PANi -coated fabric is only slightly dependent on the humidity and temperature. Therefore, it can be concluded that the effect of the temperature and humidity on the conductivity of the strain sensor can be ignored. A more direct proof of the stability of the PANi -coated fabrics is the variation of the conductivity and sensing properties of the sensor during storage of a long time. We found that the surface resistivity of the PANi coated fabric increases from $3.5 \mathrm{~K} \mathrm{ohm} /$ square to $5 \mathrm{~K} \mathrm{ohm} /$ square after 90 days. Therefore, it can be concluded that that the flexible strain sensor from PANi coated fabrics shows good environmental stability.

\section{CONCLUSIONS}

In summary, a polyaniline-coated fabric strain sensor featured with high strain sensitivity, good stability and large deformation has been developed. The sample was tested for change in resistance with linear extension and observed that the developed fabric strain sensor has a good gauge factor value of 0.92 , which is a good sensitivity value. The developed fabric strain sensor was attached to an Elbow sleeve for joint angle measurement and it was found that up to $90^{\circ}$ joint movement can be measured with repeatability. The PANi-coated fabric strain sensor so prepared is expected to find applications in sensing garment, wearable hardware and rehabilitation, etc.

\section{REFERENCES}

[1] De Rossi, D., Carpi, F., Lorussi, F., Mazzoldi, A., Paradiso, R., Scilingo, E.P., and Tognetti, A., 2003, "Electro active fabrics and wearable biomonitoring devices", AUTEX Research Journal, 3: 4.

[2] Van Langehove, L., 2007, "Smart textiles for medicine and healthcare: Materials, systems and applications", Wood head publishing.

[3] Scilingo, E.P., Lorussi, F., Mazzoldi, A., and De Rossi, D., 2003, "Strain-sensing fabrics for wearable kinaesthetic-like systems", IEEE Sensors Journal, 3: 4, pp.460-467.

[4] Xue, P.,Tao, X.M., Kwok, K.W., Leung, M.Y.,Yu, T.X. 2004, Electromechanical behavior of fibers coated with an electrically conductive polymer. Text. Res. J. 74, 929-936.

[5] Mattmann, C.,Clemens, F., Tröster, G. 2008, Sensor for measuring strain in textile. Sensors 2008, 8, 3719-3732.

[6] Oh K W, Hong K H \& Kim S H, 1999 ,"Electrically Conductive Textiles by in situ Polymerization of Aniline”, J Appl Polym Sci, 74 , 2094.

[7] Kannaian, T., Neelaveni, R., and Thilagavathi, G., 2011, "Development and characterisation of elastomeric tape sensor fabrics for elbow angle measurement", Indian Journal of Fibre and Textile Research, 36:4, pp. 436442 .

[8] LI,Y, Leung M.Y, Tao, X.M, Cheng, X.Y, Tsang, J, and Yuen, M.C.W, 2005, "Polypyrrole-coated conductive fabrics as a candidate for strain sensors", Journal of Materials Science, 40 , pp. 4093 - 4095.

[9] Atalay, O., Kennon, W. R., and Husain, M.D., 2013 ,"Textile-Based Weft Knitted Strain Sensors: Effect of Fabric Parameters on Sensor Properties", Sensors, 13, 11114-11127

[10] Holm, R.; Holm, E. 1967, Electric Contact Theory and Application. 4th Ed.; Springer-Verlag: New York, NY, USA. 